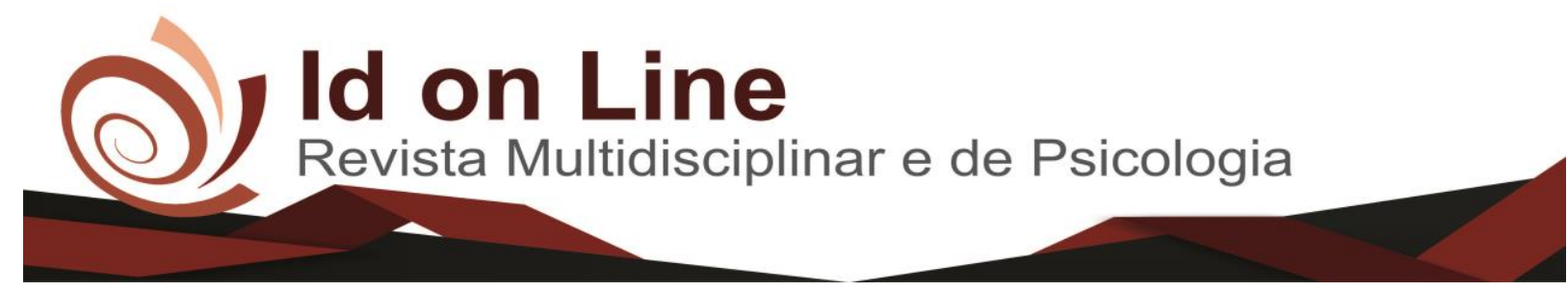

Artigo

\title{
Avaliação da Adesão ao Tratamento Farmacológico de Doenças Crônicas e Fatores Associados em Indivíduos Residentes na Zona Rural do Município de Poções-Bahia
}

\author{
Dilma Ferreira dos Santos ${ }^{l}$; Stifanny Brenda Alves dos Santos ${ }^{1}$; Larissa Morgan Andrade Lemos ${ }^{2}$.
}

\begin{abstract}
Resumo: Estudos tem demonstrado que as Doenças Crônicas Não Transmissíveis são consideradas um grande problema de saúde pública, tendo a Hipertensão e Diabetes Mellitus como agravantes para este cenário. Este estudo exploratório, com abordagem quantitativa, foi realizado em uma farmácia comunitária privada e teve como objetivo avaliar a adesão ao tratamento farmacológico da hipertensão arterial e/ou diabetes e identificar o perfil sócio demográfico, correlacionando os fatores associados em pacientes moradores da zona rural de um município do interior da Bahia. Para coleta de dados foi utilizado um questionário estruturado com questões sobre o perfil sócio demográfico, condições de saúde e uso de medicamentos. Participaram deste estudo, 250 pessoas portadores de hipertensão e/ou diabetes. Os dados apresentados neste estudo demonstram que a falta de adesão para o tratamento farmacológico das doenças crônicas pode acarretar comorbidades e, muitos outros problemas irreversíveis para a população envolvida. A adesão terapêutica poderia ser melhorada com protocolos de tratamento direcionado a cada realidade territorial.
\end{abstract}

Descritores: Hipertensão Arterial Sistêmica; Diabetes Mellitus; Adesão à farmacoterapia.

\section{Evaluation of Adhesion to Pharmacological Treatment of Chronic Diseases and Factors Associated in Indivíduos Residents in the Rural Area of the Municipality in Poções-Bahia}

\begin{abstract}
Studies have shown that Chronic no communicable Diseases are considered a major public health problem, having Hypertension and Diabetes Mellitus as an aggravating factor for this scenario. This exploratory study, with a quantitative approach was carried out in a private community pharmacy and aimed to evaluate adherence to pharmacological treatment of hypertension and/or diabetes, and identify the socio-demographic profile, correlating the associated factors in patients living in rural areas of a municipality in the interior of Bahia. For data collection, a structured questionnaire was used with questions on the socio-demographic profile, health conditions and use of medicines. 250 people with hypertension and / or diabetes participated in this study. The data presented in this study demonstrate that lack of adherence to the pharmacological treatment of chronic iseases an lead to comorbidities, and many other irreversible problems for the population involved. Therapeutic adherence could be improved with treatment protocols directed to each territorial reality.
\end{abstract}

Descriptors: Systemic Arterial Hypertension; Diabetes Mellitus; Adherence to pharmacotherapy.

\section{Introdução}

As Doenças Crônicas Não Transmissíveis (DCNT) são patologias de origem não infecciosa, com múltiplas etiologias, associadas a vários fatores de risco, com curso prolongado

${ }^{1}$ Graduanda em Farmácia Generalista pela Faculdade Independente do Nordeste. Contato: dilmafdavid@gmail.com

${ }^{2}$ Farmacêutica Clínica-industrial graduada pela Universidade Estadual de Feira de Santana. Especialista em Gestão em Saúde pela Escola Nacional de Saúde Pública Sergio Arouca da Fundação Oswaldo Cruz (ENSP/Fiocruz). Docente do curso de Farmácia na Faculdade Independente do Nordeste (FAINOR). End. Para correspondência: Rua Fernando Costa, 484 - Centro - Poções/Ba. 
e são classificadas como um grave problema de saúde pública, pois têm gerado elevado número de mortes prematuras, perda de qualidade de vida, além de impactos econômicos para famílias, comunidades e a sociedade em geral (MALTA et al., 2015).

A Organização Mundial de Saúde (OMS), fez uma estimativa em 2008 sobre a ocorrência de mais de 35 milhões de óbitos, sendo que cerca de 60\% destes, ocorreram por DCNT (WHO, 2011). Essas doenças são consideradas a principal causa de mortalidade no Brasil, resultantes de fatores e condicionantes sociais, além de fatores de risco individuais como: consumo excessivo de álcool, tabagismo, aumento do consumo de alimentação industrializada, consumo excessivo de sal e somado à tudo isso, a falta de atividade física regular (DUNCAN et al., 2012).

A hipertensão arterial sistêmica é conhecida como um dos maiores problemas de saúde pública, podendo ser considerada um importante fator de risco para o desenvolvimento de outras doenças do aparelho circulatório. Desta forma, a HAS pode ser responsável por cerca de $40 \%$ das mortes por acidente vascular cerebral, mais de $20 \%$ de mortes por doença arterial coronariana e combinada com a diabetes, é responsável por cerca de $50 \%$ dos casos de insuficiência renal terminal. A prevalência na população brasileira adulta em área urbana, varia de $22 \%$ a $43 \%$ (NEGREIROS et al., 2016).

De acordo com a Sociedade Brasileira de Diabetes (SBD), citado por Silva et al, (2016), no início deste século, uma grande parcela de todos os óbitos que ocorriam no mundo era atribuída ao diabetes, desta forma, prevê-se que em 2030, o Brasil terá uma população de aproximadamente 11 milhões de pessoas com diabetes.

O controle da hipertensão arterial sistêmica e da diabetes está fortemente associado a utilização de estratégias e abordagens que identifiquem com maior precisão os indivíduos em situação de risco, que requerem de acompanhamento e tratamento por toda a vida, envolvendo medidas farmacológicas e não farmacológicas (RADOVANOVIC et al., 2014).

Segundo Girotto et al, (2013) a adesão ao tratamento farmacológico, é um dos fatores mais importantes no controle das doenças crônicas não transmissíveis. Esta adesão pode ser considerada como a extensão pela qual o comportamento de uma pessoa reflete mudanças significativas na vida e, está diretamente associada ao cumprimento da tomada dos medicamentos, conforme a prescrição e pelo tempo determinado. Em contrapartida, a não adesão medicamentosa está relacionada à forma como o paciente conduz o tratamento, diferentemente da forma prescrita. 
Para Arruda et al, (2015) a adesão ao tratamento farmacológico pode ser compreendida quando o paciente faz uso de pelo menos $80 \%$ de todos os medicamentos prescritos, seguindo alguns fatores importantes como o horário das tomadas, a dose indicada e a duração do tratamento. Quando ocorre o uso incorreto de medicamentos, a subutilização, o uso irracional ou a não utilização dos fármacos prescritos são consideradas formas de não adesão ao tratamento medicamentoso, agravando o estágio das doenças ou acarretando em outras patologias cardiovasculares.

Estudos relatam a existência de fatores associados à não adesão ao tratamento, que estão diretamente relacionados com as características individuais de cada paciente, dentre eles, a compreensão da condição de saúde, a ausência de sinais ou sintomas que não estão relacionados apenas com a patologia, o comprometimento com o tratamento e o medicamento utilizado, incluindo a quantidade de doses diária, polifarmacoterapia, esclarecimentos sobre os serviços de saúde e o incentivo e apoio familiar (GIROTTO et al., 2013).

Não existem evidencias de estudos no Brasil, sobre a prevalência da falta de adesão para o tratamento de doenças crônicas que tenham uma real representatividade. A maioria dos estudos existentes, utilizam amostras locais ou regionais, o que dificulta o desenvolvimento de políticas públicas e outras práticas que visem melhorar esta problemática a nível nacional (REMONDI; CABRERA; SOUZA, 2014).

O Brasil tem vários programas e estratégias, destinados ao controle da hipertensão e diabetes que foram desenvolvidos objetivando promover orientações e monitoramento, com fornecimento gratuito e continuo de medicamentos e desta forma, diminuir os gastos dos usuários (COSTA et al., 2016). Entretanto, esses programas não conseguem garantir a adesão ao tratamento por esses pacientes, visto que essa adesão se trata de um fenômeno multidimensional, determinado pelo cotidiano de cada indivíduo, manifestando-se de forma particular em diferentes grupos populacionais.

Nesse contexto, objetivou-se com esse trabalho, avaliar a adesão ao tratamento farmacológico da hipertensão arterial e diabetes mellitus, correlacionando os fatores associados em pacientes residentes na zona rural do município de Poções-Bahia. 


\section{Metodologia}

Trata-se de uma investigação transversal, exploratória, com abordagem quantitativa, realizada em uma farmácia comunitária privada, localizada na região central do município de Poções-Bahia, que atende um grande fluxo de moradores da zona rural.

A amostra foi composta por 250 usuários de medicamentos, portadores de hipertensão e/ou diabetes, que buscaram a farmácia de forma espontânea no período de execução da coleta de dados. O tamanho da amostra foi calculado a partir da média do número de usuários que adquiriram seus medicamentos para o tratamento das patologias estudadas na referida farmácia nos últimos meses que antecederam a coleta de dados.

Além de portadores de hipertensão arterial e/ou diabetes, os usuários deveriam ser moradores da zona rural do município, possuir idade superior a 18 anos e não apresentar declínio cognitivo.

A coleta de dados foi realizada entre os meses de agosto e setembro de 2017, durante todos os dias da semana, onde ocorreram as entrevistas com uma prévia explicação sobre os objetivos do estudo.

Foi utilizado um questionário estruturado, que engloba dados sócio demográficos, aspectos clínicos como comorbidades, fatores de risco, medicamentos em uso, aquisição destes e a regularidade na utilização diária dos medicamentos conforme a prescrição médica, baseado nos critérios propostos por Morisky, Green e Levine (1986).

Os questionários podem ser autoaplicáveis, porém, optou-se pela entrevista, devido à possíveis dificuldades de leitura ou compreensão dos itens descritos, além da maior fragilidade que os idosos possuem na acuidade visual e na escrita. Todos os participantes da pesquisa assinaram o Termo de Consentimento Livre e Esclarecido.

Os dados coletados, foram tabulados no programa Google Drive e Microsoft Excel. A análise foi realizada com o auxílio do programa estatístico SPSS® 24.4, mediante aplicação de análise estatística descritiva.

O projeto foi desenvolvido dentro dos padrões éticos, aprovado pelo Comitê de Ética em Pesquisa - CEP da Faculdade Independente do Nordeste, de acordo com diretrizes da Resolução nº 466/2012 do Conselho Nacional de Saúde e Resolução n 510/2016, sob o CAAE $\mathrm{N}^{\mathrm{o}}$ 71193417.2.0000.5578, que aprovam diretrizes e normas regulamentadoras de pesquisas envolvendo seres humanos. 


\section{Resultados e Discussão}

Foram entrevistados 250 usuários da farmácia pesquisada, dentre estes, a idade variou entre 40 e 83 anos, com média de idade de 64 anos, houve o predomínio do sexo feminino, com ensino fundamental incompleto, casados e com renda familiar mensal entre 1 e 2 salários mínimos*3 . Quanto à ocupação, $56 \%$ realizam trabalhos rurais (Tabela 1).

Tabela 1- Resumo dos dados Sócio demográficos dos participantes da pesquisa.

\begin{tabular}{|c|c|c|}
\hline Dados Sócio demográficos & $\mathrm{N}$ & $\%$ \\
\hline \multicolumn{3}{|l|}{ SEXO } \\
\hline Feminino & 148 & 59,2 \\
\hline Masculino & 102 & 40,8 \\
\hline \multicolumn{3}{|l|}{ IDADE } \\
\hline$<60$ anos & 159 & 63,6 \\
\hline$>60$ anos & 91 & 36,4 \\
\hline \multicolumn{3}{|l|}{ ESCOLARIDADE } \\
\hline Não alfabetizado & 50 & 20,0 \\
\hline Ensino Fundamental Incompleto & 130 & 52,0 \\
\hline Ensino Fundamental Completo & 15 & 6,0 \\
\hline Ensino Médio Incompleto & 07 & 2,8 \\
\hline Ensino Médio Completo & 31 & 12,4 \\
\hline Ensino Superior Incompleto & 01 & 0,4 \\
\hline Ensino Superior Completo & 08 & 3,2 \\
\hline Técnico & 08 & 3.2 \\
\hline \multicolumn{3}{|l|}{ ESTADO CIVIL } \\
\hline Casado (a) & 171 & 68,7 \\
\hline Viúvo (a) & 53 & 21,3 \\
\hline Divorciado (a) & 16 & 6,4 \\
\hline Solteiro (a) & 10 & 4,0 \\
\hline \multicolumn{3}{|l|}{ OCUPAÇÃO } \\
\hline Trabalhador Rural & 96 & 38,4 \\
\hline Aposentado & 83 & 33,2 \\
\hline Dona de Casa & 50 & 20,0 \\
\hline Outra Atividade & 21 & 8,8 \\
\hline \multicolumn{3}{|l|}{ RENDA FAMILIAR } \\
\hline Até 1 salário mínimo & 91 & 36,4 \\
\hline 1 a 2 salários mínimos & 149 & 59,6 \\
\hline Acima de 2 salários mínimos & 10 & 4,4 \\
\hline
\end{tabular}

*Valor do salário Mínimo em 2017 durante pesquisa, R\$ 937,00 (Novecentos e trinta e sete reais). Fonte: dados da pesquisa 2017. 
A população feminina neste estudo, foi representada por 59,2\%, esse dado pode estar relacionado ao fato evidenciado por Bertoldi et al. (2016), Silva; Fonseca; Rossi (2015) e Araujo et al. (2016), em seus estudos relatam que as mulheres fazem mais uso de medicamentos e procuram mais os serviços de saúde em busca de tratamento que os homens, por isso, estão mais presentes em estabelecimento de saúde.

O uso de medicamentos para doenças crônicas por pessoas mais velhas, como foi verificado nesse estudo, corrobora com a pesquisa realizada por Ramos et al, (2016) que através de entrevistas aplicadas em cinco regiões brasileiras, verificou que os idosos apresentam ao menos uma doença crônica e, em consequência desse fator, frequentam mais os serviços de saúde em busca de medicamentos.

Quando interrogados sobre as possíveis doenças crônicas que apresentavam, 40,8 \% dos participantes da pesquisa revelaram possuir hipertensão arterial sistêmica, seguido de 5,2 \% que apresentam diabetes mellitus e 14,4\% relataram ter HAS e diabetes mellitus associadas (Tabela 2). A HAS, segundo a OMS, citado por Lobo et al, (2017) está relacionada a exposição à comportamentos de risco, como maus hábitos alimentares, tabagismo, além das dificuldades de acesso aos serviços de saúde).

A hipertensão arterial e diabetes mellitus associadas, que neste estudo foram citadas por 14,4\% dos entrevistados, são consideradas pelo Ministério da Saúde como sendo os principais agravos das doenças cardiovasculares que poderiam ser tratadas na atenção básica. Essa associação de comorbidades é bastante comum na população idosa, elevando o número de óbitos por doenças cardiovasculares (SARDINHA et al, 2015; ARAUJO et al, 2016).

Tabela 2 - Distribuição dos participantes da pesquisa de acordo com a ocorrência de DCNT.

\begin{tabular}{lcc}
\hline Doenças Apresentadas Pelos Participantes Da Pesquisa & $\mathbf{N}$ & $\%$ \\
\hline Hipertensão & 102 & 40,8 \\
Diabetes & 13 & 5,2 \\
Hipertensão e Diabetes & 36 & 14,4 \\
Diabetes e dislipidemias & 17 & 6,8 \\
Hipertensão e dislipidemias & 27 & 10,8 \\
Hipertensão e Diabetes e outra DCV & 55 & 22 \\
\hline TOTAL & 250 & 100 \\
\hline
\end{tabular}

Fonte: dados da pesquisa 2017. 
Ao serem questionados sobre a ocorrência de fatores de risco para DCNT, o sedentarismo foi o fator de risco mais frequente, com percentual de $61 \%$, seguido pelo tabagismo que apresentou frequência de 50\%. Este estudo apresentou resultado superior a estudos realizados por Silva et al, 2017 no Sul do Brasil, onde observou-se que cerca de 38\% dos entrevistados, relataram tabagismo. Fator de risco de grande preocupação para os portadores de doenças cardiovasculares, principalmente quando as populações entrevistadas em sua maioria são idosas.

Em um estudo realizado por Pegorari et al, (2015), foi revelado que a maioria dos moradores da zona rural acreditam que os esforços físicos gerados pelas atividades no campo, sejam suficientes para a saúde, sendo desnecessário a prática de outros tipos de atividade física. Esta informação pode auxiliar a compreensão do fato do sedentarismo ter sido identificado como principal fator de risco na população estudada. Além disso, sabe-se que os programas de promoção a saúde associados a atividade física são disponibilizados nos centros urbanos, não abrangendo as comunidades rurais.

No presente estudo $75 \%$ dos entrevistados relataram adquirir seus medicamentos em farmácias públicas e privadas, conforme a disponibilidade dos medicamentos nos programas governamentais. Este resultado vai de encontro a outros estudos onde foi verificado que o acesso aos medicamentos muitas vezes ocorre através de aquisição por desembolso direto, podendo gerar altos custos para os indivíduos, o que muitas vezes, compromete a renda familiar (SARDINHA et al, 2015).

A falta de informação sobre a disponibilização de medicamento por programas públicos também é um fator importante que compromete o acesso a esses produtos entre os moradores da zona rural. Esses resultados estão em conformidade com estudos que sugerem que o acesso poderia ser facilitado com políticas públicas mais eficazes para essa população específica (ARAUJO et al, 2016).

Os medicamentos mais utilizados citados pelos participantes deste estudo foram: losartana potássica, hidroclorotiazida, cloridrato de metformina e glibenclamida. Esse resultado corrobora com os dados encontrados por Sardinha et al, (2015) em um estudo realizado na zona urbana na capital do Maranhão.

Quanto ao questionamento sobre a quantidade de medicamentos utilizados diariamente, $72 \%$ dos entrevistados relataram tomar mais de um medicamento por dia, em diferentes horários. Em uma pesquisa realizada em Unidades de Saúde da Família no Ceará, a utilização diária de medicamentos por idosos variou entre dois e cinco itens distintos. $\mathrm{O}$ uso de inúmeros 
medicamentos pode ser um fator de risco para os idosos, devido a incapacidade de gerenciar os horários das tomadas, bem como as quantidades ingeridas (BEZERRA, BRITO E COSTA, 2016).

Ao avaliar a adesão com base no teste de Morisky; Green; Levine (1986), 58\% dos entrevistados relataram ter problemas para lembrar de tomar os medicamentos onde, apenas $40 \%$ dos entrevistados utilizam seus medicamentos no horário prescrito e $68 \%$ afirmou que algumas vezes esquecem de tomar os seus medicamentos. No estudo realizado por Morsch et al, (2015) no Sul do Brasil, 33\% dos entrevistados foram classificados como não aderentes quando responderam que se esquecem de tomar seus medicamentos regularmente.

Fatores como reações adversas, citado por $31 \%$ dos entrevistados, pode ser uma causa importante para o não uso dos medicamentos sem avisar ao médico. 54\% relataram que esquecem de levar os medicamentos quando viajam. 51\% já pararam com o tratamento farmacológico por sentirem melhor dos sintomas apresentados pelas patologias, e 18\% relataram sentir algum incomodo, mesmo seguindo o tratamento conforme prescrito. Esses fatores também foram relevantes em estudos realizados por Tavares et al, (2016) na população brasileira urbana, onde foi encontrado baixa adesão ao tratamento para as doenças crônicas não transmissíveis.

Em relação à rotina do tratamento farmacológico, $38 \%$ dos entrevistados relataram que às vezes se esquecem de tomar os medicamentos, $20 \%$ sempre esquecem e $30 \%$ referiram quase nunca se esquecem de fazer uso dos medicamentos. Dos 250 entrevistados, 99,2\% relatou alguma vez ter problemas para lembrar de tomar os medicamentos por algum motivo (Figura $1)$.

Os principais motivos alegados para a não adesão ao tratamento farmacológico, citados em um estudo realizado por Girotto et al, (2013) no Paraná, foram o esquecimento da tomada dos medicamentos, citados por $20 \%$ dos entrevistados no presente estudo. Essas revelações permitem compreender a necessidade de adaptação às mudanças de comportamento dos portadores de doenças crônicas.

Para Silva et al, (2015) a ausência de sintomas dificulta o seguimento do tratamento farmacológico. No mesmo estudo, é apontado que cerca de $75 \%$ dos portadores de diabetes mellitus não cumprem a tomada dos medicamentos conforme prescritos. Desta forma, o controle da doença fica comprometido e os riscos de comorbidades aumentam nestes pacientes. 
Figura 1- Distribuição dos participantes da pesquisa de acordo com a ocorrência de problemas para recordar sobre a administração do medicamento.

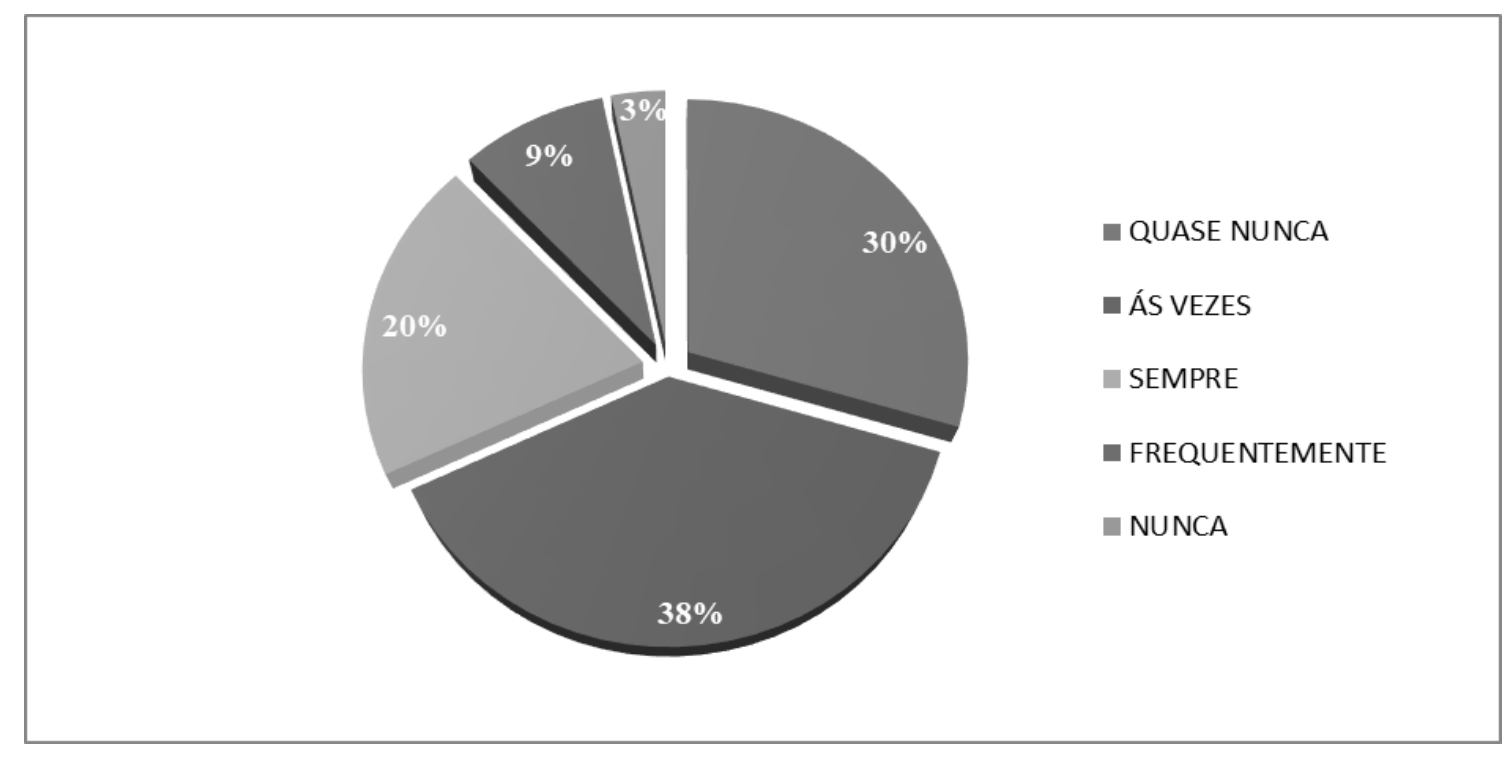

Fonte: Dados da pesquisa 2017.

Em um estudo realizado por Tavares et al, (2016) foi identificado que a falta da adesão ao tratamento farmacológico está relacionada com baixo índice de escolaridade e analfabetismo. O presente estudo encontrou dados semelhantes, pois verificou um índice de baixa escolaridade superior a 50\%, conforme mostra a Tabela 1, e o comprometimento da adesão, como demonstrado na figura 1.

Analisar as características sócio demográficas dos indivíduos, pode contribuir para identificar outros fatores importantes e relevantes para comprometer a adesão ao tratamento farmacológico e não farmacológicos de doenças crônicas não transmissíveis, que requerem tratamento longo e mudanças importantes no estilo de vida dos indivíduos e de seus familiares.

\section{Conclusão}

O estudo apresenta algumas limitações pois, ao utilizar o autorrelato para analisar o nível de adesão e identificar fatores relacionados à terapia medicamentosa, pode-se encontrar dados relatados, não condizentes com a realidade.

A falta de adesão deve ser motivo de preocupação de gestores e de toda a população envolvida, pois o uso irregular dos medicamentos aliados a fatores como alimentação 
inadequada, sedentarismo e tabagismo, tornam essa população, mais suscetíveis a comorbidades e dificuldades ainda maiores ou até irreversíveis para toda vida.

Desenvolver uma atenção diferenciada, de acordo com a realidade de cada comunidade, poderia possibilitar uma melhora no quadro de adesão terapêutica e melhor compreensão dos números encontrados em vários estudos. Além disso, protocolos de tratamento poderiam ser adaptados às comunidades rurais, considerando a realidade territorial e a necessidade de acompanhamento rotineiro nos cuidados da saúde dessa população.

\section{Referências}

ARAUJO, M. C. F., et al. Perfil de Não Adesão ao Tratamento de Usuários com Diabetes e Hipertensão em uma Unidade de Saúde da Família. Ensaios Cienc. Cienc. Biol. Agrar. Saúde, v.20, n.1, p.43-48, 2016.

ARRUDA, D. C. J. et al. Fatores associados a não adesão medicamentosa entre idosos de um ambulatório filantrópico do Espírito Santo. Rev. Bras. Geriatr. Gerontol. Rio de Janeiro, v. 18, n. 2, p. 327-337, 2015.

BERTOLDI, A.D., et al. Utilização de medicamentos genéricos na população brasileira: uma avaliação da PNAUM 2014. Rev Saude Publica. v. 50, suppl 2:11s, 2016.

BEZERRA, T. A; BRITO, M. A. A; COSTA, K. N. F. M. Caracterização do uso de medicamentos entre idosos atendidos em uma unidade básica de saúde da família. Rev.

Cogitare Enferm. v. 21, n.1, p. 01-11, 2016

COSTA, K. S. et al. Obtenção de medicamentos para hipertensão e diabetes no Programa Farmácia Popular do Brasil: resultados da Pesquisa Nacional de Saúde, 2013. Epidemiol. Serv. Saúde [online]. v.25, n.1, p.33-44, 2016.

DUNCAN, B. B. et al. Doenças Crônicas Não Transmissíveis no Brasil: prioridade para enfrentamento e investigação. Rev Saúde Pública. São Paulo, v.46, (Supl):126-34, 2012.

GIROTTO, E. et al. Adesão ao tratamento farmacológico e não farmacológico e fatores associados na atenção primária da hipertensão arterial. Ciênc. Saúde coletiva, Rio de Janeiro, v.18, n.6, 2013.

LOBO, L. A. C., et al. Tendência temporal da prevalência de hipertensão arterial sistêmica no Brasil. Cad. Saúde Pública; vol. 33, n.6, 2017. 
MALTA, D. C. et al. A vigilância e o monitoramento das principais doenças crônicas não transmissíveis no Brasil - Pesquisa Nacional de Saúde, 2013. Rev. bras. epidemiol. [online]. v.18, n.2, p.3-16, 2015.

MORISKY, D.E.; GREEN, L.W.; LEVINE, D.M. Concurrent and predictive validity of a self-reported measure of medication adherence. Medical Care, v. 24, n. 1, p. 67-74, 1986.

MORSCH, L M. et al. Complexidade da farmacoterapia em idosos atendidos em uma farmácia básica no Sul do Brasil. Rev. Infarma Ciencias farmacêuticas. v. 27, ed. 4. p.239$247,2015$.

NEGREIROS, R. V. et al. Importância do programa hiperdia na adesão ao tratamento medicamentoso e dietetico em uma unidade de saúde da família (usf). Revista da Universidade Vale do Rio Verde, Três Corações, v. 14, n. 2, p. 403-411, 2016.

PEGORARI, M. S., et al. Prática de atividade física no lazer entre idosos de área rural: condições de saúde e qualidade de vida. Rev. Educ. Fís/UEM. vol. 26, n. 2, p. 233-241, 2015.

RADOVANOVIC, C. A. T., et al. Hipertensão arterial e outros fatores de risco associados às doenças cardiovasculares em adultos. Rev. Latino-Am. Enfermagem. Ribeirão Preto. V. 22, n. 4, p.547-53, 2014

RAMOS, L. R., et al. Polifarmácia e polimorbilidade em idosos no Brasil: um desafio de saúde pública. Rev Saúde Publica. São Paulo, v.50, n. 2, p. 9, 2016.

REMONDI, F. A.; CABRERA, M. A. S.; SOUZA, R. K. T. Não adesão ao tratamento medicamentoso contínuo: prevalência e determinantes em adultos de 40 anos e mais. Cadernos de Saúde Pública, Rio de Janeiro, v. 30 n. 1, p. 126-136, 2014.

SARDINHA, A. H. L., et al. Adesão dos idosos com doenças crônicas ao tratamento medicamentoso. Rev Pesq Saúde, v. 16, n.3, p. 154-158, 2015.

SILVA, A. B. et al. Prevalência de diabetes mellitus e adesão medicamentosa em idosos da Estratégia Saúde da Família de Porto Alegre/RS. Cad. Saúde Colet., Rio de Janeiro, v.24, n. 3, p.308-316, 2016.

SILVA, A. L., FONSECA, G. S. S., ROSSI, V. E. C. Adesão ao tratamento medicamentoso entre pessoas com diabetes mellitus. Ciência et Praxis. Passos, v. 8, n. 16, 2015. 
SILVA, A. P. A. et al. Adesão ao tratamento medicamentoso e capacidade para o autocuidado de pacientes com hipertensão arterial. Arq. Ciência Saúde. São José do Rio Preto, v. 23, n. 2. p. 76-80, 2016.

SILVA, E. F. et al. Consumo de álcool e tabaco: fator de risco para doença cardiovascular em população idosa do sul do Brasil. Revista Saúde e Desenvolvimento Humano. Canoas, v. 5, n. 1, p. 23-33, 2017.

TAVARES, N. U. L., et al. Fatores associados à baixa adesão ao tratamento farmacológico de doenças crônicas no Brasil. Rev Saude Publica. São Paulo, v. 50, n. 2, 10s, 2016.

TAVARES M. S. T., et al. Qualidade de vida e adesão ao tratamento farmacológico entre idosos hipertensos. Rev Bras Enferm. Brasília, v. 69, n.1. p. 134-41, 2016.

WORLD HEALTH ORGANIZATION. Global status report on non-communicable diseases 2010 [Internet]. Geneva: WHO, p. 176, 2011.

\section{Como citar este artigo (Formato ABNT):}

SANTOS, Dilma F. dos; SANTOS, Stifanny B.A. dos; LEMOS, Larissa M.A. Avaliação da Adesão ao Tratamento Farmacológico de Doenças Crônicas e Fatores Associados em Indivíduos Residentes na Zona Rural do Município de Poções-Bahia. Id on Line Revista Multidisciplinar e de Psicologia, 2017, vol.11, n.38, p. 468-479. ISSN: 1981-1179.

Recebido: 01.10.2017

Aceito: 03.11.2017 